\title{
Two-Photon Interferometry for High-Resolution Imaging
}

\author{
Dmitry V. Strekalov and Jonathan P. Dowling. \\ Quantum Computing Technologies Group, \\ Jet Propulsion Laboratory, California Institute of Technology, \\ MS 300-123, 4800 Oak Grove Drive, Pasadena, CA 91109. \\ Dmitry.V.Strekalov@jpl.nasa.gov
}

October 25, 2018

\begin{abstract}
We discuss the advantages of using non-classical states of light for two aspects of optical imaging: the creation of microscopic images on photosensitive substrates, which constitutes the foundation for optical lithography, and the imaging of microscopic objects. In both cases, the classical resolution limit given by the Rayleigh criterion is approximately half of the optical wavelength. It has been shown, however, that by using multi-photon quantum states of the light field, and a multi-photon sensitive material or detector, this limit can be surpassed. We give a rigorous quantum mechanical treatment of this problem, address some particularly widespread misconceptions, and discuss turning quantum imaging into a practical technology.
\end{abstract}

The idea of overcoming the limits of classical optical imaging by using multi-photon processes is fairly well known. For example, Marlan Scully discusses, in his book [1], a two-photon microscope scheme that beats the diffraction limitation by a factor of $\sqrt{2}$, by making a $\operatorname{sinc}^{4}(k x)$ diffraction pattern instead of the usual $\operatorname{sinc}^{2}(k x)$. Such narrowing of a diffraction pattern can be observed by a detector sensitive to the square of intensity, instead of just intensity itself. In other words, one needs a two-photon process to observe the $\sqrt{2}$ narrowing beyond the diffraction limit, even within classical optics. Moreover, using detectors based on a higher-order multi-photon process, which are sensitive exclusively to the higher orders of intensity, one could see even narrower diffraction patterns.

This approach would not work so well for holographic imaging used in lithography. In this technique, the desired image is composed of interference fringes of different spatial frequencies, so the resolution is given by the highest spatial frequency. This spatial frequency is equal to the inverse of the fringe period, which cannot be shorter than one half of the 
optical wavelength. It is easy to see that this period is the same for any power of intensity, e.g. a $\sin ^{4}(k x)$ fringe has the same period as a $\sin ^{2}(k x)$ fringe.

Different approaches have been suggested to obtain an interference fringe of the square of the intensity with a shorter period. It has been proposed, for example, that frequency

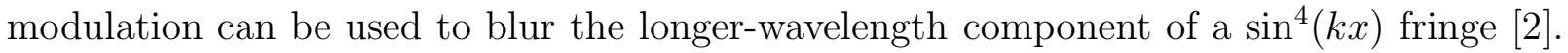
The use of quantum sources of light to beat this limit has also been proposed [3] and demonstrated with electronic coincidence detection [4].

Consider the setup in Fig.1 that has been proposed for quantum interferometric lithography [3]. This is a modification of a well-known two-photon interference experiment [5, 6], in which the single-photon detectors are removed and the output beams are directed at a two-photon sensitive substrate (e.g., one covered with a lithographical photoresist).

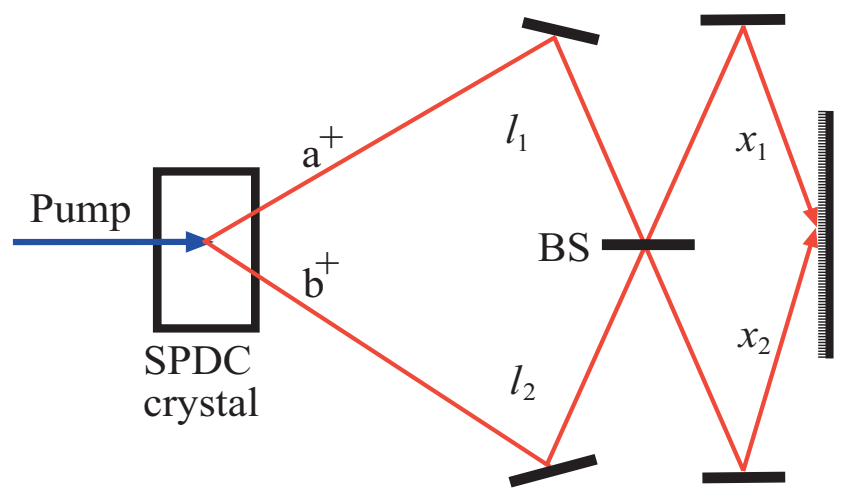

Figure 1: Two-photon interferometer with photosensitive substrate.

Following the standard theoretical treatment for two-photon interferometers, we write the two-photon square amplitude as

$$
|A|^{2} \equiv\left\langle\Psi\left|\hat{E}^{(-)} \hat{E}^{(-)} \hat{E}^{(+)} \hat{E}^{(+)}\right| \Psi\right\rangle=\left|\left\langle 0\left|\hat{E}^{(+)} \hat{E}^{(+)}\right| \Psi\right\rangle\right|^{2},
$$

where the fields depend on the propagation paths, and the state $|\Psi\rangle$ is the frequencyentangled output state of a Spontaneous Parametric Down Converter (SPDC):

$$
|\Psi\rangle=\int d \nu h(\nu) \hat{a}^{\dagger}(\nu) \hat{b}^{\dagger}(-\nu)|0\rangle
$$

In (2), creation operators $\hat{a}^{\dagger}$ and $\hat{b}^{\dagger}$ refer to channels labeled $l_{1}$ and $l_{2}$, respectively, in Fig.11; $\nu$ is the frequency-detuning from the central frequency $\omega_{0}$, the later being equal to one half of the pump frequency $\omega_{p}$. The spectral function $h(\nu)$ gives the phase-matching width and accounts for inexact momentum conservation due to the finite length $L$ of the crystal:

$$
h(\nu)=\frac{1-e^{-i L \Delta_{z}(\nu)}}{i L \Delta_{z}(\nu)} .
$$


Derivation and analysis of expressions (2) and (3) are given in a number of publications on SPDC. In particular, in [7, 8], it is shown that for collinear degenerate type-I SPDC

$$
\Delta_{z}(\nu)=-D^{\prime} \nu^{2}, \quad D^{\prime}=\left.\frac{d}{d \omega} \frac{1}{v}\right|_{\omega_{0}},
$$

and for collinear degenerate type-II SPDC, where the signal and idler photons have orthogonal polarizations, we have

$$
\Delta_{z}(\nu)=D \nu, \quad D=\frac{1}{v_{o}}-\frac{1}{v_{e}}
$$

where $v$ denotes the group velocity of the signal and idler photons. In case of orthogonal polarizations (type-II), the group velocity $v$ has indices $o$ and $e$ for "ordinary" and "extraordinary" polarization components.

The two-photon amplitude of Eq.(回) can describe the coincidence detection rate, as well as the two-photon absorption rate, as a function of pathlengths $l_{1,2}$ and $x_{1,2}$. In the coincidence detection case, the fields in Eq. (1) are evaluated at the two distinct locations of the two detectors, while in the two-photon absorption case they are evaluated at the same, arbitrary, point on the photosensitive substrate. A geometric size of the "point" in this context may be equal to the size of photo emulsion grain, or of the photoresist molecule. It is reasonable to assume that this size is much smaller than the interference structure we are expecting to see. As a further simplification, we will consider a one-dimensional problem with exactly counterpropagating beams. This geometry is obviously not practical, since no light energy is delivered to the surface, and we study this case just as an illustration allowing us to simplify the treatment.

As a next step, we need to represent the fields in (1) in terms of the same operators that describe the two-photon wavefunction, Eq. (2). For perfectly monochromatic plane waves with a wavenumber $k=\omega_{0} / c$, the representation is obtained by propagating the operators through the interferometer:

$$
\hat{E}^{(+)}=\hat{a} e^{i k l_{1}}\left(\frac{1}{\sqrt{2}} e^{i k x_{2}}+\frac{i}{\sqrt{2}} e^{i k x_{1}}\right)+\hat{b} e^{i k l_{2}}\left(\frac{1}{\sqrt{2}} e^{i k x_{1}}+\frac{i}{\sqrt{2}} e^{i k x_{2}}\right) .
$$

In equation (6), we put the proportionality constant between the field operator and the annihilation operator equal to unity. Also, we assume that the fields in the arms $l_{1}$ and $l_{2}$ have the same polarization. It is easy to see that otherwise there will be no two-photon interference fringes on the photosensitive substrate.

The plane-wave approximation implies that in the wave function Eq. (2), $h(\nu)$ should be replaced by $\delta(\nu)$. Then substituting Eqs. (6) and (2) into Eq. (11) it is easy to notice that the terms with $\hat{a}^{2}$ and $\hat{b}^{2}$ drop out, which is consistent with only one photon being present in each channel. The other four terms can be represented by four paths shown in Fig.2. These paths correspond to both photons being transmitted by the beamsplitter (a), both reflected by it (b), one transmitted, the other reflected (c), and vice versa (d). 

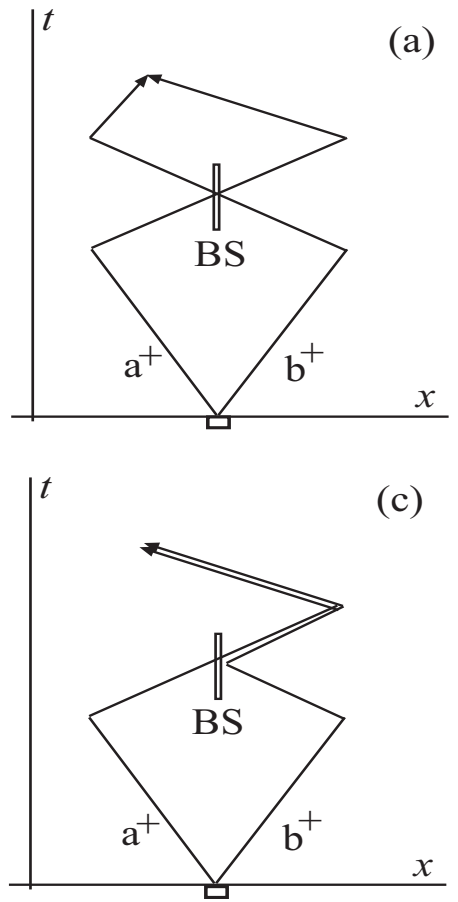
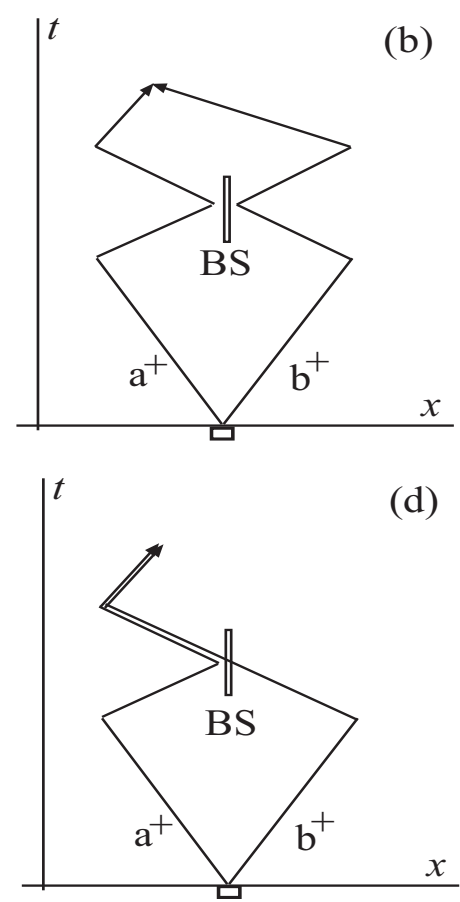

Figure 2: Different two-photon paths contributing to the amplitude (11): (a) both photons are transmitted; (b) both reflected; (c) transmitted - reflected; (d) reflected - transmitted.

Notice that, in the usual coincidence-detection treatment of two-photon interference, the amplitudes corresponding to paths (c) and (d) are discarded simply because they do not result in a pair of coincident detections. Therefore one cannot directly apply to our system the results well known for a two-detectors experiment, and then argue that the detectors are placed at the same point, since this leads to loss of the amplitudes (c) and (d). Let us now show that it is these amplitudes that give rise to two-photon interference.

In the following, we will consider the more realistic model of wavepackets rather than plane waves. The fields will be allowed to have a finite frequency bandwidth around the central frequency $\omega_{0}$, described by a real, even function $f(\nu)$ :

$$
E=\int d \nu f(\nu)\left\{\hat{a}(\nu) e^{i k(\nu) l_{1}}\left(e^{i k(\nu) x_{2}}+i e^{i k(\nu) x_{1}}\right)+\hat{b}(\nu) e^{i k(\nu) l_{2}}\left(e^{i k(\nu) x_{1}}+i e^{i k(\nu) x_{2}}\right)\right\} .
$$

Then the two-photon amplitude, Eq. (四), takes on the following form:

$$
\begin{aligned}
A= & \int d \nu d \nu_{1} d \nu_{2} h(\nu) f\left(\nu_{1}\right) f\left(\nu_{2}\right) \\
& \left\{e^{i k\left(\nu_{1}\right) l_{1}} e^{i k\left(\nu_{2}\right) l_{2}}\left(e^{i k\left(\nu_{1}\right) x_{2}}+i e^{i k\left(\nu_{1}\right) x_{1}}\right)\left(e^{i k\left(\nu_{2}\right) x_{1}}+i e^{i k\left(\nu_{2}\right) x_{2}}\right)\right\} \\
& \left\langle 0\left|\hat{a}\left(\nu_{1}\right) \hat{b}\left(\nu_{2}\right) \hat{a}^{\dagger}(\nu) \hat{b}^{\dagger}(-\nu)\right| 0\right\rangle .
\end{aligned}
$$


The inner product in Eq. (\$) is equal to $\delta\left(\nu_{1}-\nu\right) \delta\left(\nu_{2}+\nu\right)$ which reduces Eq. (8) to a single integral. To handle it, we expand $k(\nu)=k_{0}+\nu / c$, where $k_{0} \equiv k\left(\omega_{0}\right)$. This allows us to arrive at,

$$
A=e^{i k_{0}(l+x)}\left[u(\Delta l+\Delta x)-u(\Delta l-\Delta x)+2 u(\Delta l) \cos \left(2 k_{0} \Delta x\right)\right]
$$

where $u(z)$ is given by a Fourier transformation of a combined spectral density, and therefore has a meaning of a correlation function, namely,

$$
u(z) \equiv \int d \nu h(\nu) f^{2}(\nu) e^{i \frac{\nu}{c} z}
$$

In Eq. (10), the variables $x \equiv x_{2}+x_{1}, \Delta x \equiv \frac{1}{2}\left(x_{2}-x_{1}\right), l \equiv l_{2}+l_{1}$ and $\Delta l \equiv l_{2}-l_{1}$ have been introduced. Note that coordinate along the substrate $\Delta x$ is equal to a half of the path difference $x_{2}-x_{1}$.

Analyzing the symmetry properties of $h(\nu)$, we find that in both cases of type-I, Eq. (田), and type-II, Eq. (河), SPDC $u(z)$ is always a real, even function:

$$
u(z)=u(-z)=u^{*}(z)=u^{*}(-z)
$$

Therefore the first two terms in Eq. (9) cancel each other when $\Delta l=0$. Taking the absolute square of the remaining term, we get

$$
|A|^{2}=4 u^{2}(0) \cos ^{2}\left(2 k_{0} \Delta x\right) .
$$

We see from Eq. (12) that the two-photon absorption amplitude is a perioic function of coordinate $\Delta x$ measured along the photosensitive substrate, that has a spatial frequency $4 k_{0}$, which is twice the spatial frequency of the usual, second-order interference fringes. The two-photon interference fringes of Eq. (12) appear to have a perfect contrast for all $\Delta x$. This is a consequence of a plane-wave approximation for the pump. If one considers the pump with a finite bandwidth, the exponential pre-factor in (9) will no longer be just a phase factor, but will turn into an envelope, equivalent to the pump envelope. Therefore the two-photon interference fringes (12) will have a coherence length equal to the pump coherence length, which may be quite long and can reach meters for cw lasers.

It is very important that the two-photon coherence length does not depend on the bandwidth of the fields given by $f(\nu)$, nor on the phase-matching width given by $h(\nu)$. This is obvious from the condition $\Delta l=0$. It has been shown [5, 6], that in this case the two-photon amplitudes represented in Fig.2 by diagrams (a) and (b) exactly cancel each other, and the photon pair always goes to one channel (either $x_{1}$ or $x_{2}$ ), depicted in diagrams (c) or (d). In other words [3], the beamsplitter produces an entangled state, $|2\rangle_{x_{1}}|0\rangle_{x_{2}}-|0\rangle_{x_{1}}|2\rangle_{x_{2}}$, which picks up spatial phase at the same rate as the pump photon would. It also dephases at the same slow rate as the pump photon does, due to its finite bandwidth, which results in the two-photon coherence length of the SPDC light being equal to the pump (single-photon) coherence length. 
Now let us consider the linear interference in our apparatus. This analysis is important, since the modulations of intensity will directly affect the result Eq. (12) for the two-photon absorption rate. For example, there will be no two-photon absorption in the nodes of the single-photon interference fringe.

The expression for intensity is

$$
I=\left\langle\Psi\left|\hat{E}^{(-)} \hat{E}^{(+)}\right| \Psi\right\rangle,
$$

where the state $|\Psi\rangle$ is given by Eq. (2) and the field is given by Eq. (7). Setting $l_{1}=l_{2}$, and treating this expression the same way we have treated the forth-order field momenta, we arrive at

$$
I=1-\cos \left(2 k_{0} \Delta x\right) \int d \nu|h(\nu)|^{2} f^{2}(\nu) \sin \left(2 \frac{\nu}{c} \Delta x\right) .
$$

Notice that the integrand in Eq. (14) is an odd function, and hence the whole integral is zero and Eq. (14) equals unity. This means that in our apparatus there will be no intensity modulations due to the second-order interference, regardless of individual coherence length of the signal and idler photons. This at first appears surprising, since one might expect to see at least a few interference fringes at the white light interference condition $x_{1}=x_{2}$. However, taking into account that both inputs of the beamsplitter are used, we realize that we actually have two sets of interference fringes exactly out of phase with each other, and hence the total intensity is unmodulated.

Two more issues associated with two-photon quantum imaging need to be addressed to make it a practically useful technology. One is the availability of two-photon sensitive photoresists and detectors, and the other has to do with the fact that using SPDC as a two-photon source, one first loses a factor of two in spatial resolution by down converting the pump frequency (and hence doubling the wavelength), and then re-gains this factor by using two-photon processes. Therefore, in terms of spatial resolution, our quantum imaging technique has no apparent advantage over using classical imaging at the pump wavelength. The counter is that it is not always possible to use the UV light argument [9]. For example, it may be incompatible with imaging biological or other light sensitive objects. Another example is 3D lithography [9]. Creating 3D structures with single-photon exposure of photolithographical materials is very difficult, since they strongly absorb UV light, which limits the depth of penetration. Two-photon exposures solve this problem. However, much value would be added to the quantum imaging technology if one could prepare two-photon states without doubling the wavelength. One way to achieve it is to use a Hyper Parametric Scattering (HPS) instead of SPDC.

HPS is a nonlinear optical process occurring via the cubical optical nonlinearity $\chi^{(3)}$, in which two pump photons recombine into an entangled photon pair. This process is similar to four wave mixing in the same sense as SPDC is similar to Parametric Amplification: four wave mixing and PA assume non-vacuum input into the signal or the idler modes. HPS is distinct from the SPDC, where a single pump photon produces an entangled pair. This distinction is most evident from comparing the phase-matching conditions for SPDC 
with those for HPS:

$$
\begin{aligned}
\vec{k}_{p} & =\vec{k}_{s}+\vec{k}_{i}, & \omega_{p}=\omega_{s}+\omega_{i}, \\
2 \vec{k}_{p} & =\vec{k}_{s}+\vec{k}_{i}, & 2 \omega_{p}=\omega_{s}+\omega_{i},
\end{aligned}
$$

which is illustrated graphically in Fig. 3. An important thing to notice in Fig. 3 is that the average wavelength of the photons produced in HPS is the same as that of the pump, while in the case of SPDC it doubles.
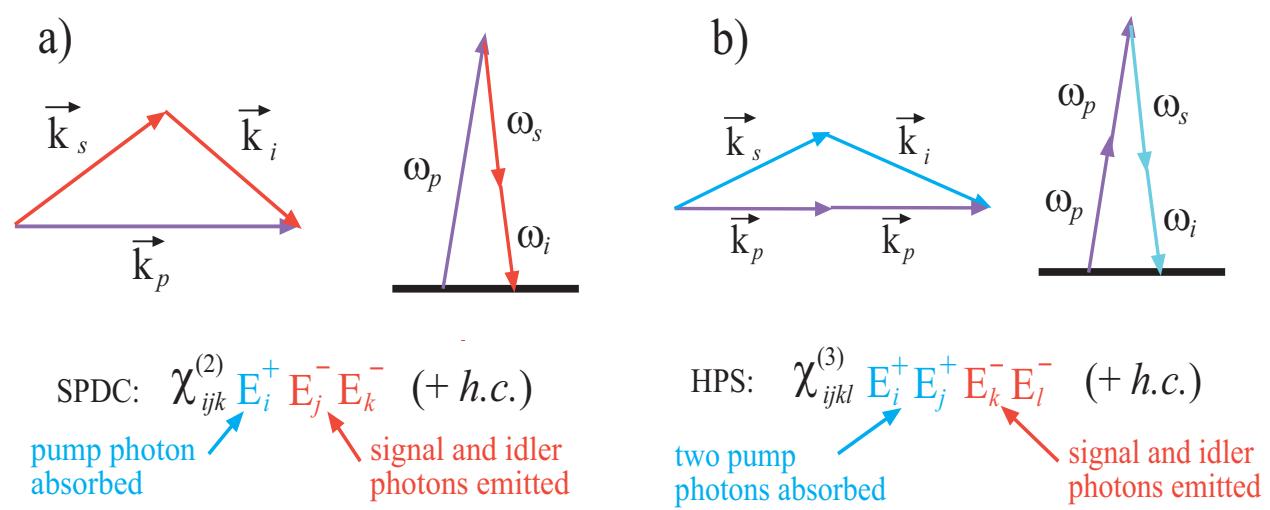

Figure 3: The phase-matching (momentum and energy conservation) diagrams for SPDC (a) and HPS (b).

HPS was observed for the first time over 30 years ago [10]. At that time, it did not attract attention as a source of EPR-states because of a very low efficiency of the $\chi^{(3)}$ processes compared to $\chi^{(2)}$ processes. A typical value for $\chi^{(2)}$ is $10^{-8}$ [CGS units of electric field $]^{-1}$, while for $\chi^{(3)}$ it is $10^{-15}$ [CGS units of field] ${ }^{-2}$. Fortunately, HPS output power is quadratic with respect to the pump intensity, while in case of SPDC it is only linear. To compare efficiencies of the two processes, one must compare the $\left|E_{p} \chi^{(2)}\right|$ and $\left|\chi^{(3)}\right|$. Modern powerful femtosecond lasers, that were not yet available in the early days of HPS, dramatically changed the situation in favor of HPS.

Another argument in favor of HPS is that, unlike SPDC, this process does not require any particular symmetry of the media and can be observed not only in crystals but also in glass fibers [11], which promises to increase the interaction length to meters or beyond. Furthermore, it has been shown [12] that nearly four orders of magnitude improvement of the signal can be achieved by cascading two $\chi^{(2)}$ processes to emulate a $\chi^{(3)}$ HPS process. A large amount of research has been done on $\chi^{(3)}$ processes, and particularly on four wave mixing [11,13 15], and we plan to rely on these results in our new research program directed at creating a robust source of entangled photon pairs or two-photon states without down converting from a higher frequency.

The second practical issue, mentioned above, is the availability of two-photon sensitive photoresists. Considering the very low power of two-photon sources, a high two-photon 
sensitivity of the photoresists is required. Unfortunately, high, single-photon, UV sensitivity of many commercially available photoresists does not guarantee that they would be suitable two-photon sensitive materials. The synthesis of such a material appears to be a difficult task, although a large volume of research has been done in this area motivated by the growing recognition of the two-photon imaging technology importance [9, 16, 17].

We also have carried out a preliminary search for two-photon sensitive lithographic materials. Relying on the analogy with atomic systems, we expect that a suitable twophoton material would have an intermediate level corresponding to the single photon energy, so that the single-photon detuning, which factors inversely into the two-photon absorption cross section, is small, and the two-photon absorption rate is peaked. It is furthermore required that the molecular transition corresponding to the intermediate absorption level does not result in the photochemical reaction in the phototresist (otherwise the resist would be one-photon sensitive). Finally, we require that the intermediate level or band is normally depopulated and very short-lived (otherwise the resist would be one-photon sensitive via cascaded processes); and that both transitions have the correct selection rules.

We have taken absorption spectra of various commercially available photoresists. The results are shown in Fig. 4 . One of our samples, the Novalac 5740, has shown a local absorption maximum which is centered at about $520 \mathrm{~nm}$ and is clearly separated from the strong transition in the UV part of the spectra, which is associated with the photochemical reaction initiating the photoresist. We spun an approximately $15 \mu \mathrm{m}$-thick sample of this photoresist on a gold plated substrate and exposed the sample to different doses of the Argon Ion laser light, whose wavelength $(514.5 \mathrm{~nm})$ was close to the center of the absorption peak of interest. We found the threshold dose of about $2 \mathrm{~kJ} / \mathrm{cm}^{2}$, assuming $100 \%$ radiation reflection off the mirror substrate and operating at the intensity level of 5 $\mathrm{W} / \mathrm{cm}^{2}$. Repeating the experiment at $25 \mathrm{~W} / \mathrm{cm}^{2}$, we obtained the same results with an exposure time that was five times shorter. This result suggests that the exposure process is linear in intensity and hence is a single-photon one. Notice that the threshold we found at $514.5 \mathrm{~nm}$ is roughly five orders of magnitude higher then for a regular UV exposure.

Next, we repeated the exposures for another Argon Ion laser line with the $457.9 \mathrm{~nm}$ wavelength, which is off the intermediate absorption peak but is closer to the UV absorption transition. We found that at this wavelength the threshold dose was definitely lower than $0.4 \mathrm{~kJ} / \mathrm{cm}^{2}$. This suggests that the high-threshold photo-initiation observed at $514.5 \mathrm{~nm}$, as well as at $457.9 \mathrm{~nm}$, is not related to the intermediate absorption peak, but rather is due to a far off-resonant absorption on the wing of the UV absorbing transition. Therefore, the selected material may satisfy the above-outlined requirements for a two-photon optimized photoresist, and it would be interesting to try exposing it with a two-photon source. We plan on carrying out such experiment in the nearest future.

In conclusion, we have carried out a rigorous analysis that confirmed the earlier results [3]. In addition, our analysis has shown that the desired two-photon interference fringe will have a very long coherence length, equal to that of the pump, and that the secondorder (single-photon) interference fringes will be entirely absent. The questions related to 


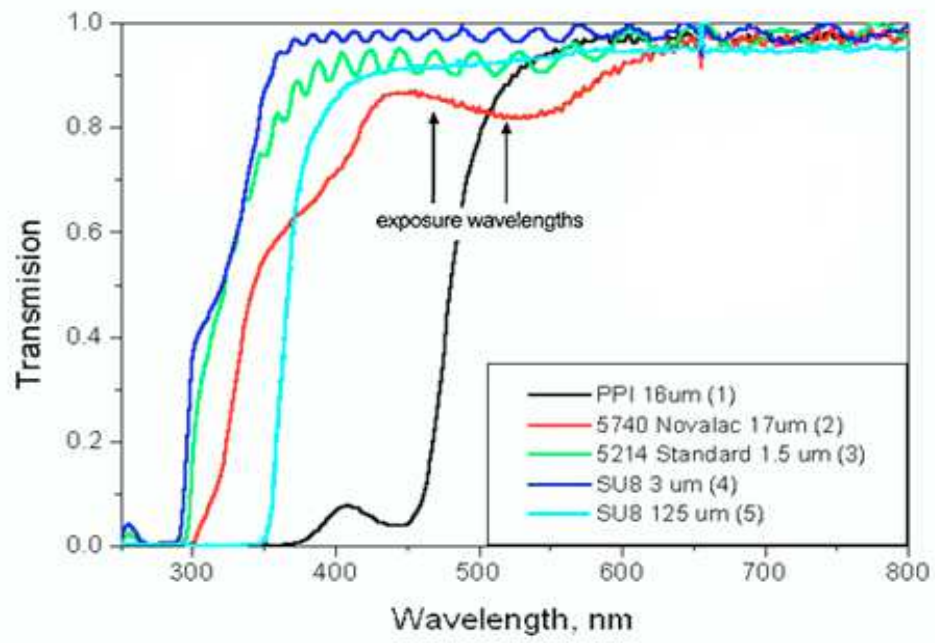

Figure 4: Absorption spectra for different photoresists. The sample of the choice shows an absorption maximum centered at about $520 \mathrm{~nm}$. Arrows mark the wavelengths the sample was exposed at: $457.9 \mathrm{~nm}$ and $514.5 \mathrm{~nm}$.

alternative sources of two-photon states and to the choice of two-photon sensitive photolithographical materials have been discussed. Although bringing the research in this area to the level of practical technology is a challenging task, it is at the same time is an interesting and potentially rewarding one.

\section{Acknowledgments}

We would like to acknowledge useful discussions with R. Y. Chiao, J. D. Franson, D. F. V. James P. G. Kwiat, W.D. Phillips, Y. H. Shih, J. E. Sipe, and A. M. Steinberg. We thank Victor White for valuable help with photoresists. We would like to acknowledge financial support from the National Aeronautics and Space Administration, the Office of Naval Research, and the Advanced Research and Development Activity.

\section{References}

[1] M.O. Scully and M.S. Zubairy. Quantum Optics. Cambridge University Press, Cambridge UK, 1997.

[2] E. Yablonovitch and R.B. Vrijen. Opt. Eng., 38:334, (1999).

[3] A.N. Boto, P. Kok, D.S. Abrams, S.L. Braunstein, C. P. Williams, and J.P. Dowling. Quantum interferometric optical lithography: exploiting entanglement to beat diffraction limit. Phys. Rev. Lett., 85:2733-36, (2000).

[4] M. D'Angelo, M.V. Chekhova, and Y.H. Shih. Two-photon diffraction and quantum lithography. To appear in Phys. Rev. Lett., , (2001). 
[5] C.K. Hong, Z.Y. Ou, and L. Mandel. Measurement of subpicosecond time intervals between two photons by interference. Phys. Rev. Lett., 59:2044-2046, (1987).

[6] Y.H. Shih and C.O. Alley. New type of Einstein-Podolsky-Rosen experiment using pairs of light quanta produced by optical parametric down conversion. Phys. Rev. Lett., 61 :2921-2924, (1988).

[7] Y.H. Shih, A.V. Sergienko, M.H. Rubin, T.E. Kiess, and C.O. Alley. Two-photon entanglement in type-II parametric down-conversion. Phys. Rev. A, 50:23-28, (1994).

[8] A.V. Burlakov, M.V. Chekhova, D.N. Klyshko, S.P. Kulik, A.N. Penin, Y.H. Shih, and D.V. Strekalov. Interference effects in spontaneous two-photon parametric scattering from two macroscopic regions. Physical Review A, 56:3214-25, 1997.

[9] K.D. Belfield, K.J Schafter, Y. Liu, J. Liu, X. Ren, and E.W. Van Stryland. J. of Phys. Org. Chem., 13:837-849, (2000).

[10] D.L. Weinberg. Appl. Phys. Lett., 16:32, (1969).

[11] P. Kumar and M.I. Kolobov. Opt. Comm., 104:374, (1994).

[12] D.N. Klyshko. Photons and Non-linear Optics. Gordon and Breach Science, New York, 1988.

[13] R.E. Slusher and et al. Phys. Rev. Lett., 55:2409, (1985).

[14] E.M. Nagasako, R.W. Boyd, and G.S. Agarwal. Phys. Rev. A, 55:1412, (1997).

[15] R.W. Boyd and G.S. Agarwal. Phys. Rev. A, 59:R2587, (1999).

[16] R.A. Borisov and et al. Appl. Phys. B, 67:765, (1998).

[17] M.P. Joshi, H.E. Pudavar, J. Swiatkievicz, P.N. Prasad, and B.A. Reinhardt. Appl. Phys. Lett., 74:170, (1999). 